'Departamento de Anatomía Patológica, Facultad de Medicina, Pontificia Universidad Católica de Chile. Santiago, Chile. 2Departamento de Oncología, Facultad de Medicina, Pontificia Universidad Católica de Chile. Santiago, Chile.

Este trabajo no recibió financiamiento; los autores declaran no tener conflictos de interés.

Recibido el 28 de noviembre de 2018, aceptado el 2 de diciembre de 2019.

Correspondencia a: Dr. Gonzalo P. Méndez.

Departamento de Anatomía Patológica, Facultad de Medicina, Pontificia Universidad Católica de Chile, Santiago, Chile. Marcoleta 377, Piso 7, Santiago 8330024 , RM, Chile. gmendez@med.puc.cl

\section{Correlación anátomo-clínica entre clases y subclases proliferativas de nefritis lúpica en biopsias renales: experiencia de un centro único}

\author{
LORETO BREVIS ${ }^{1}$, SERGIO VARGAS ${ }^{2}$, \\ DAVID ODDÓ ${ }^{1}$, GONZALO P. MÉNDEZ ${ }^{1}$
}

\section{Clinicopathological correlation among proliferative classes and subclasses of lupus nephritis in renal biopsies}

Background: Lupus nephritis (LN) is a complication of systemic lupus erythematosus that requires renal biopsy (RB). Proliferative classes III, IV-S, $I V-G$ have especial clinical and pathological characteristics. Aim: To determine the association between pathological features in $R B$ with serum creatinine and urine protein levels. Material and Methods: We analyzed 186 RB performed in adults aged 18 to 73 years, from a renal pathology reference center. Histopathological variables such as class and subclass of proliferative LN, endocapillary and extracapillary proliferation, activity and chronicity indexes, and vascular sclerosis were correlated with serum creatinine and urine protein levels, at the time of diagnosis. Results: As compared with LN III, all the morphological and laboratory values were significantly more deteriorated in LNIV, with special focus on vascular sclerosis. Serum creatinine was the only variable that significantly differentiated LNIV-S from LNIV-G. Proteinuria was non-significantly higher in LN IV-G compared to LN IV-S. However, the difference became significant when proteinuria was compared between LN IV-G and LN III. Conclusions: The significant difference in serum creatinine between $L N I V-S$ and $L N I V-G$ supports the concept that they are different subclasses. Proteinuria is a variable that differentiates classes III from IV-G, being significantly higher in the second. Severe arteriosclerosis is a constant and significant finding that differentiates $L N$ III from LN IV. Thus, we propose its usefulness for distinguishing LN classes, and eventually, to be considered in the chronicity index.

(Rev Med Chile 2019; 147: 1510-1517)

Key words: Biopsy; Creatinine; Kidney; Lupus Erythematosus, Systemic; Lupus Nephritis; Proteinuria.
$\mathrm{E}$ 1 lupus eritematoso sistémico es una enfermedad autoinmune de origen desconocido y multifactorial ${ }^{1,2}$, con prevalencia de $20-150$ casos por 100.000 habitantes $^{3}$ e incidencia de 1-25 por 100.00 habitantes ${ }^{1,4}$. Un $65 \%$ debuta entre los 16 y 55 años $; 20 \%$ en menores de 16 años $^{6}$.
La nefritis lúpica (NL) es una manifestación muy grave, con prevalencia de $50 \%{ }^{3}$. Si no se trata oportunamente, puede causar insuficiencia renal terminal y muerte ${ }^{3,7}$. Si bien es debatible su indicación, ante un sedimento urinario activo, proteinuria, hematuria, insuficiencia renal, resis- 
tencia al tratamiento o recaídas, se recomienda realizar biopsia renal (BR), ya que puede orientar el tratamiento y establecer pronóstico según sus hallazgos ${ }^{2,7-9}$.

La NL se clasifica según patrones histopatológicos de lesión glomerular. Los criterios establecidos en el año $2003^{10}$ determinan seis clases: I (morfología "normal”); II (mesangial); III (proliferativa intracapilar y/o extracapilar focal, o sea, $<50 \%$ del total de glomérulos afectados); IV (proliferativa intracapilar y/o extracapilar difusa, o sea, $>50 \%$ del total de glomérulos afectados); $\mathrm{V}$ (membranosa) y IV (esclerosante; > 90\% de glomérulos esclerosados). Las clases proliferativas son las clases III (focal) y IV (difusa), subdividiendo clásicamente esta última, en segmentaria (IV-S) y global (IV-G), dependiendo respectivamente si las lesiones que predominan en más de $50 \%$ de los glomérulos afectan a una parte de su ovillo capilar o son más bien completas.

Sorpresivamente, existen pocas publicaciones de correlación de variables histopatológicas con laboratorio, en pacientes con NL proliferativa ${ }^{11,12}$. Esta carencia de información es particularmente notoria en nuestro país, donde no existen registros de glomerulopatías. La literatura internacional muestra resultados disímiles en cuanto a correlación clínico-patológica entre las subclases IV-G y IV-S, y entre las clases III y IV de NL. Esta información se asume muy valiosa para análisis que validen y establezcan modificaciones en su clasificación, incluyendo a la reciente actualización ${ }^{13-17}$.

Considerando que las clases III y IV de NL presentan diferencias en intensidad y extensión de sus lesiones inflamatorias glomerulares así como en su presentación clínica, este estudio tiene como objetivo definir dentro de nuestra experiencia local, el perfil de presentación morfológico y de laboratorio urinario de las NL clases III y IV, de subclases IV-G y IV-S, y describir sus eventuales diferencias o similitudes en estos aspectos.

\section{Pacientes y Método}

Estudio observacional, descriptivo y retrospectivo, de muestras de BR con diagnóstico de NL clase III y IV (ISN/RPS), en pacientes hombres y mujeres, adultos y pediátricos (menores de 17 años), examinadas y diagnosticadas en el Departamento de Anatomía Patológica de la
Facultad de Medicina de la Pontificia Universidad Católica de Chile, entre enero de 2008 y diciembre de 2015.

La información de edad, sexo y valores de laboratorio fueron obtenidos desde las solicitudes de biopsias renales recibidas. Las variables de laboratorio utilizadas en este estudio fueron exclusivamente la creatinina sérica $(\mathrm{mg} / \mathrm{dL})$ y proteinuria $(\mathrm{g} / 24 \mathrm{~h})$, ya que son los datos más consistentemente consignados en el formulario de solicitud.

Este estudio fue aprobado por el Comité de Ética en Investigación de la Escuela de Medicina de la Pontificia Universidad Católica (CEI-MedUC), con el número de proyecto: 160905001 // 16-258.

\section{Caracterización de nefritis lúpica}

Todos los casos de NL fueron examinados y diagnosticados por un nefropatólogo, en base a los criterios ISN/RPS establecidos ${ }^{10}$. En todos los casos se realizó examen de microscopía óptica, de inmunofluorescencia y microscopía electrónica de transmisión.

Para facilitar el análisis estadístico del significado de la presencia de lesiones proliferativas (endocapilares y extracapilares) en cada una de las clases y subclases de NL, se cuantificó el número de glomérulos con lesión proliferativa en relación al número total de glomérulos examinados (índice "número glomérulos con lesión/número total de glomérulos en la biopsia").

Los índices de actividad y cronicidad fueron determinados según el índice de Austin, sin considerar su reciente modificación ${ }^{11}$.

La intensidad de esclerosis vascular fue clasificada como: negativa a leve, si los vasos tenían un aspecto normal o sólo mínima presencia de material colagenoso reemplazando la íntima o media; acentuada, si los vasos tenían presencia de material colagenoso reemplazando la íntima o media en mayor extensión mural o perimetral (más de 25\%).

Se excluyeron las biopsias renales con diagnóstico de NL clases I, II, V, VI y NL mixta (III+V y $\mathrm{IV}+\mathrm{V}$ ) así como casos con menos de 7 glomérulos en microscopía óptica.

\section{Análisis estadístico}

Las variables fueron separadas en dos subgrupos:

Variables no histológicas: demográficas (sexo, 
edad) y de laboratorio (creatinina sérica y proteinuria de $24 \mathrm{~h}$ ). Se utilizaron solo estas dos variables de laboratorio urinario debido a que son las más consistentemente recibidas dentro de la información clínica de cada biopsia.

Variables histológicas: NL clase III (NL III), NL clase IV-G (NL IV-G), NL clase IV-S (NL IV-S), proliferación endocapilar, proliferación extracapilar (crecéntica), índice de actividad, índice de cronicidad y esclerosis vascular.

La distribución gaussiana de las variables continuas fue determinada con el test de normalidad omnibus de D'Agostino-Pearson. Para variables categóricas, se utilizó el test de Chi-cuadrado para analizar potenciales diferencias entre los grupos. Alternativamente, las potenciales diferencias de las variables continuas fueron analizadas con el test de Kruskall-Wallis y análisis post hoc de Dunn, dado que la distribución de la mayoría de las variables fue no gaussianas.

Para realizar una asociación multivariada entre las variables no histológicas y las histológicas, así como con clasificación de NL, se utilizaron análisis de Regresión Lineal Multinominal Lineal o de Regresión Logística Multinominal, dependiendo de la naturaleza de la variable dependiente (continua o categórica, respectivamente). Finalmente, se trazaron curvas ROC para evaluar el rendimiento estadístico del modelo de regresión, sólo para variables categóricas. Los resultados se consideraron estadísticamente significativos cuando el valor-p fue menos que 0,05 .

El análisis estadístico fue realizado en software SPSS 20.0 y los gráficos fueron trazados con GaphPad Prism 5.0.

\section{Resultados}

Se obtuvieron 186 biopsias evaluables de NL clases III y IV, separadas en dos grupos: pacientes menores de 17 años (pediátricos) y pacientes mayores de 17 años (adultos). En la Tabla 1, se resume el número y distribución de casos.

Como el grupo pediátrico no alcanzó un número mínimo de casos para realizar análisis estadísticos inferenciales, éstos se realizaron solo en el grupo de adultos. El grupo de adultos alcanzó a 159 casos. La distribución de género fue constante en las tres subclases respectivas de NL proliferativas (Figura 1A), variable que no presentó una relación de significancia estadística (p 0,8985) con los subtipos de NL.

La edad promedio para todos los hombres fue de 27,9 años (rango de 18 a 73 años) y, para las mujeres, el promedio fue de 32,7 años (rango de 17 a 67 años). El análisis reveló que la edad por cada clase de NL tampoco fue una variable estadísticamente significativa ( $p>0,05$; IC 95\%). La edad promedio por grupo fue: NL III: $33 \pm 11$ años; NL IV-S: $33 \pm 11$ años; NL IV-G: $31 \pm 11$ años.

\section{Variables de laboratorio}

La Tabla 2 muestra los subgrupos de NL cuyos pacientes tenían disponibles valores de proteinuria $(\mathrm{n}=79)$ y creatinina sérica $(\mathrm{n}=105)$.

Para la variable creatinina sérica $(\mathrm{mg} / \mathrm{dL})$, los valores promedio por grupos fueron: NL III: 0,77 $\pm 0,32$; NL IV-S: $1,19 \pm 0,86$; NL IV-G: $2,45 \pm 1,67$. Se observaron diferencias estadísticamente significativas (p 0,001) entre la NL III respecto de ambas subclases de NL IV, y también entre las NL IV-S y NL IV-G (Figura 1B).

Respecto de la variable proteinuria $(\mathrm{g} / 24 \mathrm{~h})$, el valor promedio para cada grupo fue: NL III: $2,34 \pm 2,24$; NL IV-S: $2,91 \pm 2,13$; NL IV-G: $4,52 \pm 3,38$. Se observó una diferencia estadísticamente significativa ( $\mathrm{p}$ 0,0078) entre NL III y NL IV-G. Por el contrario, no hubo diferencias entre NL III con NL IV-S, ni entre los subtipos de NL IV (Figura 1C).

Tabla 1. Distribución y número de BR de NL proliferativas según grupo etario y sexo

\begin{tabular}{|cccccccc|}
\hline & Total & Fexo & N & NL III & NL IV-S & NL IV-G \\
\hline$<17$ años & 27 & 17 & M & 10 & 13 & 6 & 8 \\
$\geq 17$ años & 159 & 150 & 9 & 47 & 46 & 66 \\
\hline
\end{tabular}



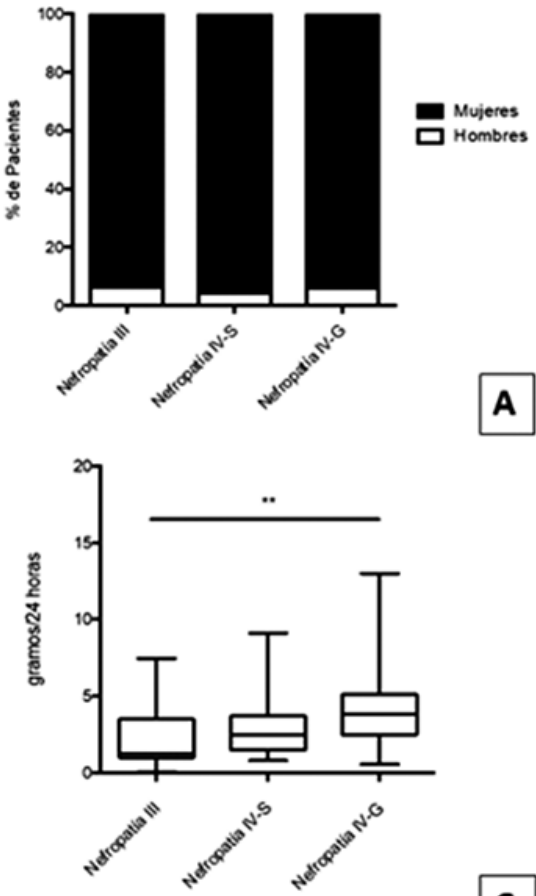

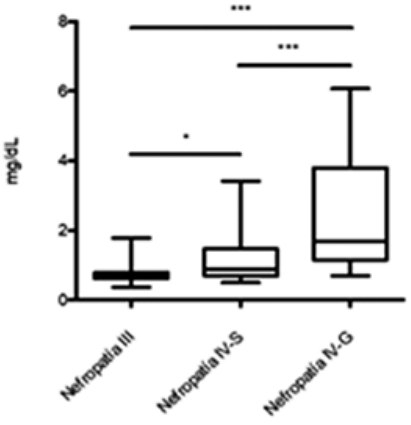

B

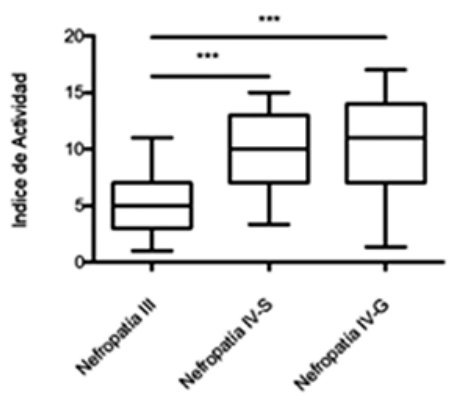

Figura 1. A. Gráfico de barras donde se observa el predominio de mujeres en cada clase de NL, siempre por sobre el $90 \%$ en cada una de ellas. B. Gráfico de cajas que muestra la relación entre los valores promedio de creatinina sérica $(\mathrm{mg} / \mathrm{dL}) \mathrm{para}$ cada grupo de NL. La línea horizontal sobre las cajas muestra un valor estadísticamente significativo ( $p<0,001$, IC 95\%) entre clases III y IV-S (señalado con *); III y IV-G (señalado con ***), así como entre los subtipos de NL IV (señalado con ${ }^{* *}$ ). C. Gráfico de cajas que correlaciona proteinuria (gramos/24 h) con los tres tipos de NL proliferativa; se observa una diferencia significativa ( p 0,0078; IC 95\%), solo entre NL III y NL IV-G (línea marcada con **). D. Gráfico de cajas que muestra el puntaje promedio del índice de actividad para de cada tipo de NL. Se observa una relación estadísticamente significativa (p 0,001; IC 95\%) en la actividad de la NL III con el grupo de NL IV-S, y entre la actividad en NL III con NL IV-G (ambas relaciones señaladas con ***).

Tabla 2. Distribución y número de BR de NL proliferativas con información disponible en relación a proteinuria y creatinina sérica

\begin{tabular}{|lcccccc|}
\hline & \multicolumn{2}{c}{ NL III } & \multicolumn{2}{c}{ NL IV-S } & \multicolumn{2}{c|}{ NL IV-G } \\
& información & $\begin{array}{c}\text { Total } \\
\text { pacientes }\end{array}$ & $\begin{array}{c}\text { Con } \\
\text { información }\end{array}$ & $\begin{array}{c}\text { Total } \\
\text { pacientes }\end{array}$ & $\begin{array}{c}\text { Con } \\
\text { información }\end{array}$ & $\begin{array}{c}\text { Total } \\
\text { pacientes }\end{array}$ \\
\hline Proteinuria de $24 \mathrm{~h}$ & 25 & 47 & 25 & 46 & 29 & 66 \\
Creatinina sérica & 28 & & 37 & & 40 & \\
\hline
\end{tabular}

\section{Índices de actividad y cronicidad}

El puntaje promedio del índice actividad para cada grupo fue el siguiente: NL III: $5,1 \pm 3,0 ; \mathrm{NL}$ IV-S: 9,7 $\pm 3,7$; NL IV-G: $10,3 \pm 4,5$. La NL III mostró una diferencia significativa respecto de la NL IV-S y de la NL IV-G. Ambas subclases de NL
IV no presentaron diferencias (Figura 1D).

El puntaje promedio del índice de cronicidad según tipo de NL fue: NL III: $0,9 \pm 1$; NL IV-S: $1,7 \pm 2,1$; NL IV-G: $2,5 \pm 2,9$. La única diferencia significativa se presentó entre las NL III y NL IV-G (Figura 2A). 

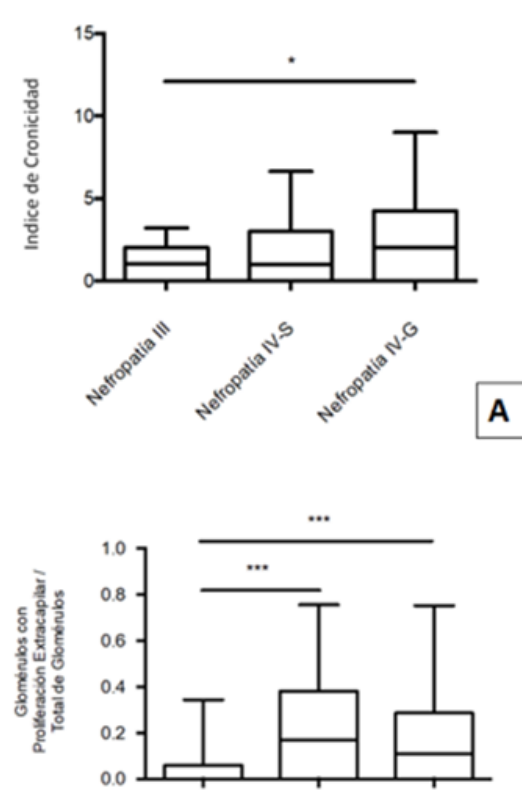

c
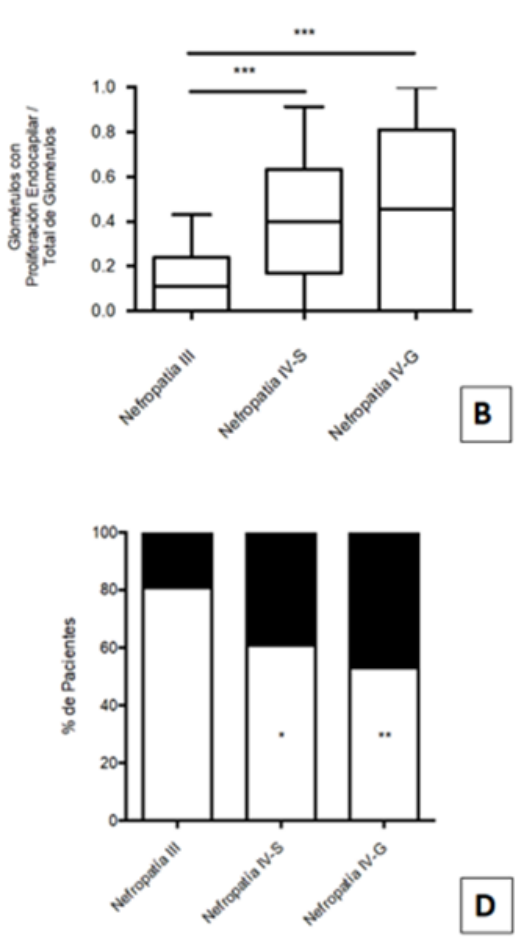

Figura 2. A. Gráfico de cajas que muestra el índice de cronicidad en cada NL. Existe una asociación con significancia estadística ( p 0,0152, IC 95\%) entre el puntaje de cronicidad del grupos NL III con NL IV-G (señalado con *), no siendo significativas las diferencias entre NL III con NL IV-S, ni entre los subtipos de NL IV. B. Gráfico de cajas que muestra el índice entre glomérulos con proliferación endocapilar y el número total de glomérulos en cada subgrupo de NL. Esta variable fue estadísticamente significativa ( $p<0,0001$, IC 95\%) entre NL III y NL IV-S, así como entre NL III con NL IV-G (ambos señalados con ***). Al comparar los subtipos de NL IV entre sí, no hubo significancia estadística $(p>0,05)$. C. Gráfico de cajas donde se observa el índice entre glomérulos con proliferación extracapilar y el número total de glomérulos en cada subgrupo de NL. Se observa que esta variable es estadísticamente significativa ( $p<0,0001$; IC 95\%) al comparar NL III respecto de la NL IV-S, y entre NL III con NL IV-G G (ambos señalados con ${ }^{* *}$ ), pero no así entre los subtipos de NL IV. D. Gráfico de barras que muestra el porcentaje de esclerosis vascular en cada subgrupo de NL. En negro, los casos considerados positivos (esclerosis vascular moderada a intensa) y en blanco los negativos (sin esclerosis o esclerosis leve). Existe una diferencia con significancia estadística ( p 0,0093; IC 95\%) para los grupos NL IV-S (señalado con *) y NL IV-G (señalado con **), respecto del grupo NL III, pero no así entre ambos subtipos de NL IV.

\section{Variables histológicas especiales (proliferación endocapilar, proliferación extracapilar, esclerosis vascular) \\ En las Figuras 2B, 2C y 2D se observan y des-} criben los resultados del análisis de estas variables en relación a cada tipo de NL.

\section{Discusión}

La BR es importante en el diagnóstico, tratamiento y pronóstico de la NL $\mathrm{NL}^{1,2,7,9,10}$, con escasas publicaciones de correlación anátomo-clínicas ${ }^{10,12}$. Esto tiene mucha relevancia en la reproducibilidad e interpretación de la histología del lupus, que muestra diferencias a veces mínimas ${ }^{9,13,18,19}$ que pueden determinar cambios en su clasificación.

Los resultados de este estudio fueron obtenidos teniendo como base un grupo de pacientes de edad adulta con diagnóstico de NL clases III y IV, y cuyo predominio de sexo femenino es consistente con la prevalencia de género conocida ${ }^{1}$.

Al estudiar la intensidad de proteinuria al momento de la BR, no se observaron diferencias 
significativas entre las subclases IV-S y IV-G, donde ambas tendieron a mostrar más proteinuria respecto de la clase III, lo que favorece una presentación y comportamiento similares de toda la NL IV según esta variable. Sin embargo, en el análisis específico, la NL IV-G presentó niveles significativamente mayores de proteinuria respecto de la NL III, lo que desde el análisis estadístico sugiere, por esta asociación, un potencial carácter más agresivo en la presentación de la NL IV-G. Este resultado no favorece unificar la NL IV, más aun considerando que la proteinuria es un factor de mal pronóstico en cualquier enfermedad renal, especialmente en $\mathrm{NL}^{20}$.

La diferencia en el nivel de proteinuria, especialmente entre los grupos IV-G y III, podría explicarse por presencia de lesiones inflamatorias más difusas para el primero, afectando potencialmente en mayor intensidad y/o extensión a las nefronas en su función y estructura de la barrera de ultrafiltración, mediado por moléculas pro inflamatorias y complemento ${ }^{21}$. De aquí se puede inferir que existe algún mecanismo "protector" en la barrera de ultrafiltración que sería sobrepasado cuando las lesiones inflamatorias se hacen más globales en cada glomérulo, lo que explicaría por qué no hubo diferencias significativas entre la subclases IV-S y III.

Existen publicaciones que muestran que la proteinuria acentuada caracteriza a la subclase IV-G al compararla con la subclase IV-S ${ }^{16,18}$. Si bien nuestros resultados no mostraron diferencias significativas entre ambas subclases, sí observamos una tendencia, donde la proteinuria del grupo IV-G prácticamente duplicó a la del grupo IV-S. Esta falta de diferencia estadísticamente significativa se debió seguramente a un $\mathrm{N}$ bajo, lo que trajo como consecuencia una mayor desviación estándar. Dado que este fue un estudio exploratorio, no se determinó un tamaño de muestras mínimo $y$, por lo tanto, sugerimos ampliar la cohorte en estudios venideros para validar estadísticamente esta diferencia.

La creatinina sérica fue la única variable que mostró diferencias significativas entre todos los subgrupos de NL entre sí, hallazgo concordante con lo descrito en la literatura y cuya asociación sugiere que a mayor grado de cambios proliferativos glomerulares, tanto en número como en superficie de glomérulos, es esperable un grado mayor de insuficiencia renal ${ }^{13,15,16,18}$. Esto puede explicarse por una progresiva disminución de la superficie de ultrafiltración glomerular, lo cual finalmente lleva a una caída de la velocidad de filtración y aumento de la creatinina sérica.

Dentro de las variables morfológicas estudiadas, nuestro estudio demuestra la utilidad de realizar la cuantificación de los índices de actividad y cronicidad, ya que estos mostraron diferencias significativas entre los distintos grupos. Esta conclusión se ve refrendada en la actual revisión de $\mathrm{NL}^{17}$. En particular, el índice de actividad fue útil para diferenciar ambas subclases de NL IV respecto de la NL III, existiendo mayor actividad cuando la nefritis es difusa que cuando es focal. Sin embargo, esta variable no mostró diferencias significativas dentro de las subclases IV.

El índice de cronicidad mostró una diferencia significativa, aunque de menor magnitud, entre la clase III y la clase IV-G, lo que plantea la posibilidad que a mayor extensión del compromiso inflamatorio, existe la tendencia de alcanzar más temprano fases cicatrizales de daño glomerular, probablemente porque un mayor grado de proliferación puede determinar una nefritis más agresiva en cuanto a lesiones destructivas, aunque siempre dependiendo de la temporalidad de la aparición del cuadro nefrítico. Esta variable tampoco mostró diferencias entre las subclases de NL IV, lo que apoya que el daño crónico es más probable en nefritis de mayor extensión y eventualmente temporalidad. Considerando que no todos los estudios utilizan estos índices, destacamos que algunas publicaciones muestran hallazgos en concordancia con los nuestros ${ }^{16,18}$.

La hipercelularidad endocapilar fue una variable morfológica que mostró diferencias en las clases de NL de nuestra cohorte. Específicamente, la proporción de glomérulos comprometidos por esta alteración tuvo directa relación con las distintas clases de NL, lo que apoya desde el análisis morfológico, la diferencia entre clases III y IV de NL. Las NL IV-S y NL IV-G si bien no mostraron diferencia estadística, la tendencia fue de mayor celularidad endocapilar para la segunda.

Del mismo modo, el compromiso por lesiones proliferativas extracapilares fue significativamente menor en la NL III, contrastando con ambas subclases de NL IV. Este hallazgo concuerda con que la presentación clínica de nefritis lúpicas difusas es más activa, aunque las publicaciones que han estudiado esta asociación son escasas, requiriendo 
estudios adicionales para confirmarla. Esta misma variable no mostró diferencias dentro de las subclases de NL IV, concordando con los principales estudios disponibles ${ }^{13,16}$, aunque publicaciones aisladas $^{15}$ plantean lo contrario. Nuevamente, consideramos indispensable contar con mayor cantidad de estudios y casos para confirmar estos resultados.

La asociación estadística de la variable esclerosis vascular fue un hallazgo interesante, especialmente porque no está considerada históricamente dentro de la clasificación de BR con NL. Nuestros resultados mostraron una diferencia significativa entre las clases III y IV ( G y S), con mayor grado de esclerosis vascular en pacientes del segundo grupo, no existiendo diferencias entre ambas subclases IV-S y IV-G. Ante este hallazgo, creemos que esta variable morfológica podría ser incorporada en el índice de cronicidad y, aún más, considerarse eventualmente como otro factor en la diferenciación entre NL III y NL IV.

Concordamos en que nuestro estudio tiene la limitación de que no se analizaron otras variables clínicas ni datos de laboratorio adicionales atingentes a NL; por lo tanto, este análisis de correlación partió desde los hallazgos morfológicos de las BR y los datos de laboratorio estudiados dependieron de la información clínica recibida en las solicitudes de biopsia respectivas. A pesar de esto último, creemos que nuestros resultados de correlación aportan a la discusión, principalmente morfológica, de si las categorías histológicas proliferativas de NL deban considerarse como entidades diferentes. En particular, nuestros resultados favorecen la existencia de subclases de NL IV, ya que muestran diferencias morfológicamente significativas que se correlacionan con las variables de laboratorio urinario estudiadas.

Finalmente, consideramos nuestros resultados, desde el análisis estadístico y morfológico, como una importante primera aproximación hacia el perfil de NL proliferativas en pacientes de nuestro medio y que puede servir de base para estudios relacionados en el futuro cercano.

\section{Referencias}

1. Schur PH, Hahn BH. Epidemiology and pathogenesis of systemic lupus erythematosus. UpToDate. Feb 05,
2016. (https://www.uptodate.com/contents/epidemiology-and-pathogenesis-of-systemic-lupus-erythematosus/ print).

2. Borchers AT, Leibushor N, Naguwa SM, Cheema GS, Shoenfeld Y, Gershwin ME. Lupus nephritis: a critical review. Autoimmun Rev 2012; 12 (2): 174-94.

3. Pons-Estel GJ, Alarcón GS, Scofield L, Reinlib L, Cooper GS. Understanding the epidemiology and progression of systemic lupus erythematosus. Semin Arthritis Rheum 2010; 39 (4): 257-68.

4. Danchenko N, Satia JA, Anthony MS. Epidemiology of systemic lupus erythematosus: a comparison of worldwide disease burden. Lupus 2006; 15 (5): 308-18.

5. Rothfield N. Clinical features of systemic lupus erythematosus. In: Textbook of Rheumatology, Kelley WN, Harris ED, Ruddy S, Sledge CB (Eds), WB Saunders, Philadelphia 1981.

6. Schaller J. Lupus in childhood. Clin Rheum Dis 1982; 8 (1): 219-28.

7. Aguirre V, Alvo M, Ardiles L, Carpio JD, Foster C, Goecke A, et al. A consensus of the Chilean Nephrology and Rheumatology Societies on renal involvement in systemic lupus erythematosus. Rev Med Chile 2015; 143 (12): 1569-78.

8. Smith PP, Gordon C. Systemic lupus erythematosus: clinical presentations. Autoimmun Rev 2010; 10 (1): 43-5.

9. Mittal B, Rennke HG, Singh AK. The role of kidney biopsy in the management of lupus nephritis. Curr Opin Nephrol Hypertens 2005; 14 (1): 1-8.

10. Weening JJ, D'Agati VD, Schwartz MM, Seshan SV, Alpers CE, Appel GB, et al. The Classification of Glomerulonephritis in Systemic Lupus Erythematosus Revisited. J Am Soc Nephrol 2004; 15 (2): 241-50.

11. Austin HA 3rd, Muenz LR, Joyce KM, Antonovych TT, Balow JE. Diffuse proliferative lupus nephritis: identification of specific pathologic features affecting renal outcome. Kidney Int 1984; 25 (4): 689-95.

12. Wilhelmus S, Alpers CE, Cook HT, Ferrario F, Fogo AB, Haas M, et al. The revisited classification of GN in SLE al 10 years: time to re-evaluate histopathologic lesions. J Am Soc Nephrol 2015; 26 (12): 2938-46.

13. Mittal B, Hurwitz S, Rennke H, Singh AK. New subcategories of class IV lupus nephritis: are there clinical, histologic, and outcome differences? Am J Kidney Dis 2004; 44 (6): 1050-9.

14. Haring CM, Rietveld A, van den Brand JA, Berden JH. Segmental and global subclasses of class IV lupus nephritis have similar renal outcomes. J Am Soc Nephrol 2012; 23 (1): 149-54.

15. Wagrowska-Danilewicz M, Danilewicz M. Clinicopa- 
thological characteristics of segmental and global active subclasses of class IV lupus nephritis: a comparative study. Pol J Pathol 2014; 65 (3): 223-8.

16. Hill GS, Delahousse M, Nochy D, Bariéty J. Class IV-S versus class IV-G lupus nephritis: clinical and morphologic differences suggesting different pathogenesis. Kidney Int 2005; 68 (5): 2288-97.

17. Bajema IM, Wilhelmus S, Alpers CE, Bruijn JA, Colvin $\mathrm{RB}$, Cook HT, et al. Revision of the International Society of Nephrology/Renal Pathology Society classification for lupus nephritis: clarification of definitions, and modified National Institutes of Health activity and chronicity indices. Kidney Int 2018; 93 (4): 789-96.
18. Nasri H, Baradaran A, Rafieian-kopaei M, Mubarak M. Significance of global versus segmental subclassification of class III and IV lupus nephritis: a single center experience. Acta Reumatol Port 2015; 40 (2): 138-44.

19. Wilhelmus S, Cook HT, Noël LH, Ferrario F, Wolterbeek R, Bruijn JA, et al. Interobserver agreement on histopathological lesions in class III or IV lupus nephritis. Clin J Am Soc Nephrol 2015; 10 (1): 47-53.

20. Hasegawa T, Suzuki K, Kaneko Y, Takeuchi T. Proteinuria selectivity index as a prognostic biomarker in lupus nephritis. Lupus 2017; 26 (6): 656-60.

21. Nagata, M. Podocyte injury and its consequences. Kidney International 2016; 89 (6): 1221-30. 